\title{
Active Multiple Plasmon-Induced Transparency with Graphene Sheets Resonators in Mid-Infrared Frequencies
}

\author{
Jicheng Wang, ${ }^{1,2}$ Baojie Tang, ${ }^{2}$ Xiushan Xia, ${ }^{2}$ and Shutian Liu ${ }^{1}$ \\ ${ }^{1}$ Department of Physics, Harbin Institute of Technology, Harbin 150001, China \\ ${ }^{2}$ School of Science, Jiangnan University, Wuxi 214122, China \\ Correspondence should be addressed to Jicheng Wang; jcwanghit@163.com and Shutian Liu; stliu@hit.edu.cn
}

Received 20 January 2016; Accepted 30 March 2016

Academic Editor: Yan Wang

Copyright (c) 2016 Jicheng Wang et al. This is an open access article distributed under the Creative Commons Attribution License, which permits unrestricted use, distribution, and reproduction in any medium, provided the original work is properly cited.

\begin{abstract}
A multiple plasmon-induced transparency (PIT) device operated in the mid-infrared region has been proposed. The designed model is comprised of one graphene ribbon as main waveguide and two narrow graphene sheets resonators. The phase coupling between two graphene resonators has been investigated. The multimode PIT resonances have been found in both cases and can be dynamically tuned via varying the chemical potential of graphene resonators without optimizing its geometric parameters. In addition, this structure can get multiple PIT effect by equipping extra two sheets on the symmetric positions of graphene waveguide. The simulation results based on finite element method (FEM) are in good agreement with the resonance theory. This work may pave new way for graphene-based thermal plasmonic devices applications.
\end{abstract}

\section{Introduction}

Surface plasmon polaritons (SPPs), coupled modes of plasmons and photons, propagate along the metal-dielectric interface $[1,2]$. SPPs provide an intriguing approach to localize and guide light in subwavelength metallic structures. In recent years, a great variety of efforts have been devoted to applications based on SPPs, which promotes the development for high density integration of photonic circuits. Electromagnetically induced transparency (EIT) is a sharp transparency window within a broad absorption spectrum in the medium, which is due to the coherent interaction between atomic upper levels and applied optical field $[3,4]$. Recently, a novel analogy phenomenon to EIT, known as plasmonically induced transparency (PIT), has been manifested in various systems based on metamaterial structures [5-12]. The PIT effect has been applied in various applications such as nonlinear optical processes, ultrafast switching, slow light, and signal processing $[13,14]$. However, the most of PIT effects are based on MIM (metal-insulator-metal) structure, and the adjustment of PIT effects must rely on changing the geometrical parameter of structures. Recently, multimode
PIT effects have been achieved by dual coupled grapheneintegrated ring resonators $[15,16]$. The MIM was replaced by graphene structure to achieve PIT effects without modifying parameter of structure. The dynamically tunable PIT planar hybrid metamaterials have been proposed, in which the $\mathrm{VO}_{2}$ stripes are filled in the grooves of the device surface. The simulation results indicate that the PIT effects can be achieved by changing the temperature of $\mathrm{VO}_{2}$ stripes [17]. To compare with [18], the structure of our proposal is simpler and more convenient in modulating PIT effects.

In recent years, graphene has aroused extensive attention for its unique and fantastic properties in optics and electronics [19]. A monolayer graphene has been widely researched as a promising platform for plasmonics for well supporting SPPs on graphene $[20,21]$. Most importantly, the surface conductivity of graphene could be dynamically tuned by chemical potential via gate voltage, electric field, magnetic field, and chemical doping [22], which increasingly promotes the development of active plasmonic devices including polarizers [23], high-speed optical modulators [24], absorbers [25], and transformation optical devices [26]. Zeng et al. have 


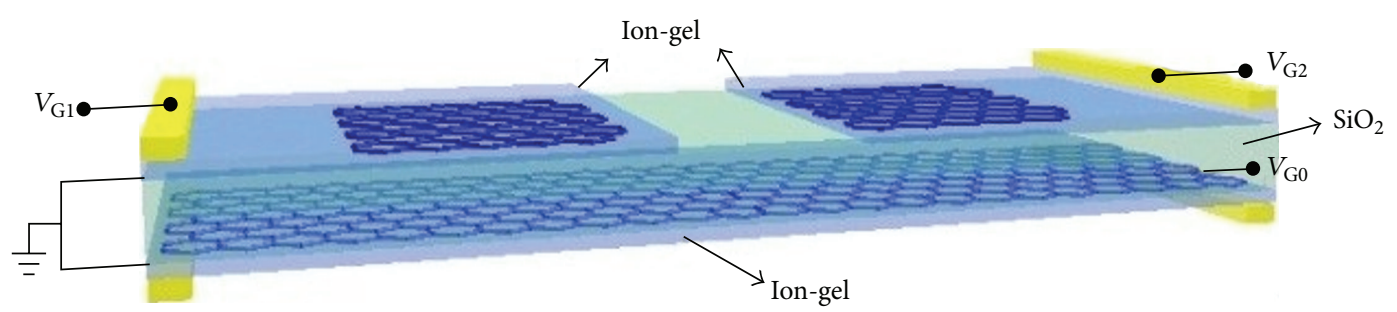

(a)

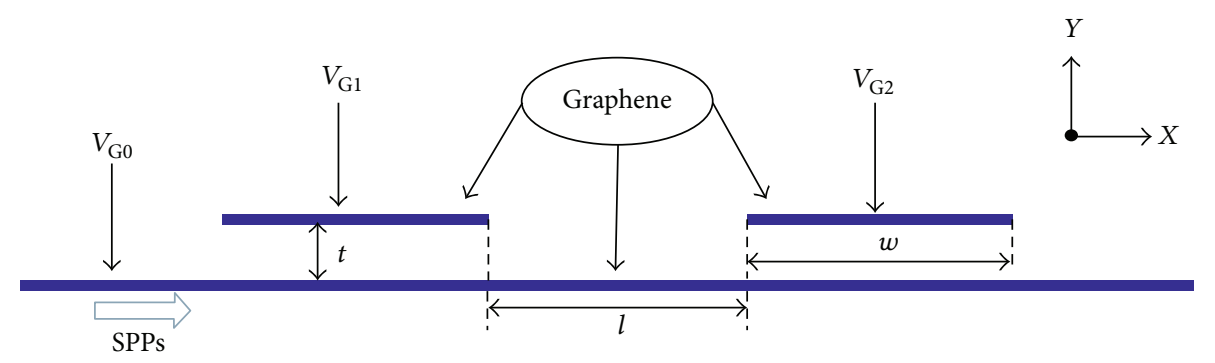

(b)

FIGURE 1: Schematic of plasmonic structure consists of two graphene ribbon and two graphene sheets. (a) Three-dimensional schematic of the plasmonic device. (b) Side view of the device configuration. $w=200 \mathrm{~nm}$ is the length of two graphene sheets; $t=50 \mathrm{~nm}$ is the coupling distance between graphene waveguide and two graphene sheets; $l=300 \mathrm{~nm}$ is the separation between two graphene sheets; $V_{\mathrm{G} 0}$, $V_{\mathrm{G} 1}$, and $V_{\mathrm{G} 2}$, respectively, represent the gate voltages on graphene waveguide and two graphene sheets.

studied a graphene-integrated Fabry-Perot (F-P) microcavity to efficiently modulate plasmon-induced transparency effect [27]. Shi et al. numerically demonstrated a plasmonic analog to EIT based on graphene nanostructures [28]. Therefore, the monolayer graphene is a promising platform to design a dynamically wavelength tunable PIT planar device.

In this paper, we have proposed and investigated a PIT device operating in the mid-infrared region. The designed model consisted of one graphene ribbon as main waveguide and two narrow graphene sheets as resonators. Firstly, a graphene waveguide side-coupled with single graphene resonator has been studied as a filter. Next, the phase coupling between two graphene resonators side-coupled to the main graphene waveguide has been investigated. In order to modulate multipeak PIT, four sheets were inserted into the symmetric position of graphene waveguide. The multimode and multipeak PIT resonances can be actively controlled via changing the chemical potential (Fermi energy) of graphene. Our designs have been well verified by numerical simulation based on finite element method (FEM). Hence, the proposed structures may have great potential in ultra-compact graphene thermal plasmonic devices in the infrared range.

\section{Method}

The structure of proposed device is schematically depicted in Figure 1. It consists of one graphene ribbon as main waveguide and two uniform graphene sheets as resonators. For experimental consideration, the designed graphene system is assumed in the $\mathrm{SiO}_{2}$ medium. It should be noted that the $\mathrm{SiO}_{2}$ medium is replaced with air in simulation for simplicity. The Kubo formula has governed the optical conductivity of graphene including the interband and intraband transition contributions [29]. It depends on the momentum relaxation time $\tau$, temperature $T$, incident wavelength $\lambda$ (angular frequency $\omega$ ), and chemical potential (Fermi energy $\left.E_{f}\right) \mu_{c}$. The surface conductivity of graphene $\sigma_{g}$ follows the equation

$$
\begin{aligned}
\sigma_{g}= & \frac{i e^{2} \mu_{c}}{\pi \hbar^{2}\left(\omega+i \tau^{-1}\right)}+\frac{i e^{2}}{4 \pi \hbar} \ln \left[\frac{2\left|\mu_{c}\right|-\left(\omega+i \tau^{-1}\right) \hbar}{2\left|\mu_{c}\right|+\left(\omega+i \tau^{-1}\right) \hbar}\right] \\
& +\frac{i e^{2} k_{B} T}{\pi \hbar^{2}\left(\omega+i \tau^{-1}\right)} 2 \ln \left[\exp \left(-\frac{\mu_{c}}{k_{B} T}\right)+1\right] .
\end{aligned}
$$

In our simulation, the employed incident light is in the mid-infrared region where the intraband transition contribution dominates in monolayer graphene [30]. Under this condition, the optical conductivity is given by

$$
\sigma_{g}(\omega)=\frac{i e^{2} \mu_{c} / \pi \hbar^{2}}{\omega+i \tau^{-1}}
$$

Here, $e$ is the electron charge and the carrier relaxation time $\tau=\mu \mu_{c} /\left(e v_{f}^{2}\right)$ relates to the carrier mobility $\mu$, chemical potential $\mu_{c}$, and Fermi velocity $v_{f}=10^{6} \mathrm{~m} / \mathrm{s}$ in graphene. The equivalent permittivity of graphene is given by the equation [26]

$$
\varepsilon_{g, e q}=1+\frac{i \sigma_{g} \eta_{0}}{k_{0} \Delta},
$$

where $k_{0}=2 \pi / \lambda$ is wave number in vacuum and $\eta_{0} \approx$ $377 \Omega$ is the intrinsic impedance of air. The carrier mobility is reasonably chosen to be $\mu=20000 \mathrm{~cm}^{2} \mathrm{~V}^{-1} \mathrm{~s}^{-1}$ from experiment results $[25,31]$. The thickness of graphene is assumed as $\Delta=0.5 \mathrm{~nm}$ in order to take the numerical 


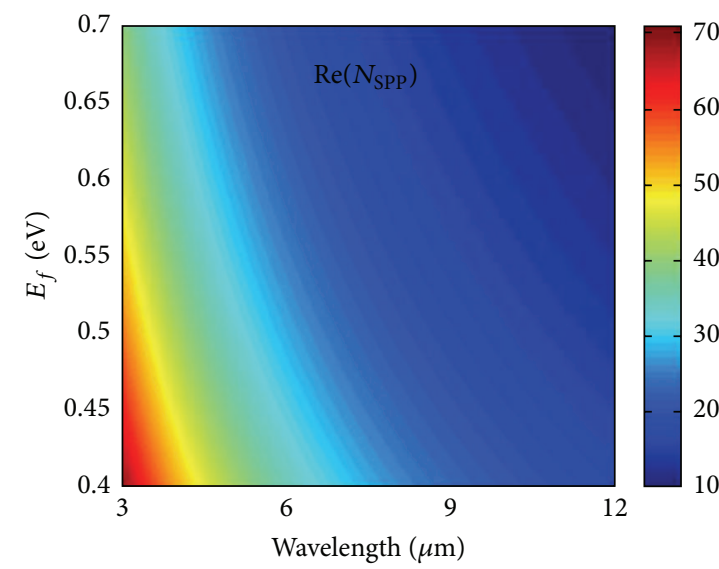

(a)

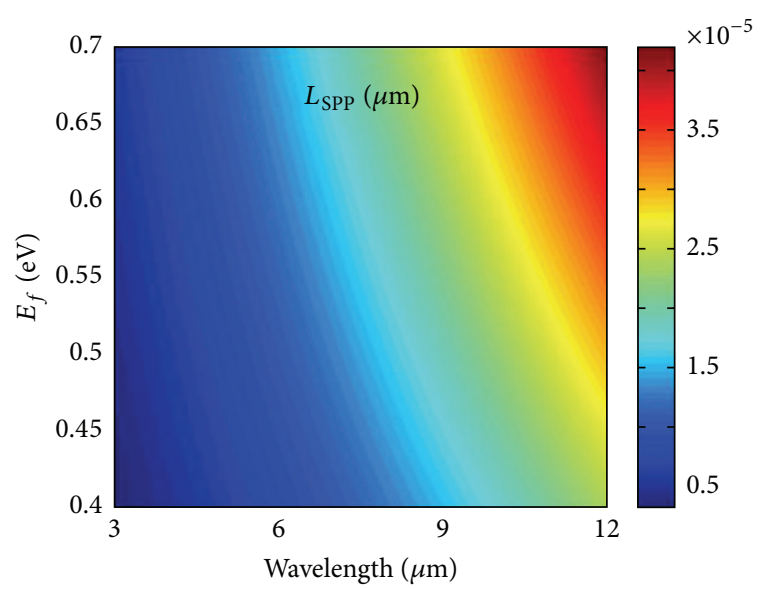

(b)

FIGURE 2: (a) Real part of effective refractive index for graphene SPPs as a function of incident wavelength and Fermi level. (b) Dependence of propagation length $L_{\mathrm{SPP}}$ on incident wavelength and the Fermi level.

simulation, but $\Delta$ is not the real thickness of the graphene [19, 21]. TM- (Transverse Magnetic-) polarized SPPs on graphene are only in consideration for the investigation. The dispersion relation of this TM SPP surface wave follows the equation

$$
\beta_{\mathrm{SPP}}=k_{0} \sqrt{\varepsilon_{r}-\left(\frac{2 \varepsilon_{r}}{\eta_{0} \sigma_{g}}\right)^{2}},
$$

where $\beta_{\text {SPP }}$ is the propagation constant of graphene SPPs in air $\left(\varepsilon_{r}=1\right)$. The effective refractive index of graphene SPPs shows the ability to confine SPPs on graphene, which is defined as $N_{\mathrm{SPP}}=\beta_{\mathrm{SPP}} / k_{0}$. The propagation length $L_{\mathrm{SPP}}=1 / \operatorname{Im}\left(\beta_{\mathrm{SPP}}\right)$ that indicates the SPP propagation loss in graphene. The dependences of $\operatorname{Re}\left(N_{\mathrm{SPP}}\right)$ and $L_{\mathrm{SPP}}$ on the Fermi level $E_{f}$ and incident light wavelength $\lambda$ are shown in Figure 2. Obviously, from Figure $2(\mathrm{a}), \operatorname{Re}\left(N_{\mathrm{SPP}}\right)$ increases as the Fermi level $E_{f}$ decreases for a fixed wavelength, which means that SPPs are better confined when Fermi level is lower. Nevertheless, the tendency in Figure 2(b) is evidently opposite to that in Figure 2(a), indicating that a lower Fermi level gives a smaller propagation length. Thus, both these important factors should be taken into consideration in the design of PIT systems. Interestingly, $\operatorname{Re}\left(N_{\mathrm{SPP}}\right)$ varies greatly when the Fermi level is changed slightly, which provides a way to actively control our configuration.

\section{Result and Discussion}

At first, we focus on one single graphene resonator sidecoupled to graphene waveguide as a filter. As shown in Figure 1, there are two graphene sheet resonators whereas we set the right resonator inactive by fixing its $E_{f}$ at $0.05 \mathrm{eV}$. Under this condition SPPs will not be supported on graphene because the interband transition of electrons occurs at this Fermi energy [11]. The Fermi level of graphene waveguide is fixed at $0.4 \mathrm{eV}$. When SPPs on graphene meets the resonant condition, the graphene resonator will influence the transmission of main waveguide by forming standing wave. The resonance wavelength is determined by the width and Fermi level of the graphene resonator, which can be deduced from quasi-static analysis and Drude-like model $[19,32]$. It follows the following equation:

$$
\lambda_{r} \approx \frac{2 \pi \hbar c}{e} \sqrt{\frac{\varepsilon_{r} \varepsilon_{0} \pi \eta \omega}{\chi E_{f}}} .
$$

Here, $c$ is the velocity of light in vacuum and $\chi$ parameter is introduced for a freestanding graphene sheet $\left(\chi=1 / \varepsilon_{r}=1\right)$ [33]. The fitting dimensionless parameter $\eta$ can be derived from numerical simulation $[19,31,34]$ and $w=200 \mathrm{~nm}$ is the width of graphene sheet. The resonance condition is given by $2 \operatorname{Re}\left(k_{\mathrm{SPP}}\right) w+\Phi=2 m \pi$ [34]. Here, $m$ is an arbitrary positive integer, resonance mode number, and $\Phi$ is an additional phase shift because of the edge ends of the graphene sheets. Actually, the influence of the edge ends is only effective when the structure is in extremely small size, so we can neglect the influence in most cases $[33,35]$. Besides, another equation for propagation constant of SPPs on monolayer graphene has been derived by combining the Drude-like model with the dispersion relation [36]:

$$
k_{\mathrm{SPP}} \approx \frac{8 \pi^{3} \hbar^{2} c \varepsilon_{r}}{e^{2} E_{f} \eta_{0} \lambda^{2}}+i \frac{4 \pi^{2} \hbar^{2} \varepsilon_{r}}{e^{2} E_{f} \eta_{0} \tau \lambda} .
$$

By using the FEM, the transmission spectra have been calculated for different Fermi level $E_{f}$ of left graphene resonator ranging from $3.6 \mu \mathrm{m}$ to $5 \mu \mathrm{m}$, shown in Figure 3(a). The above discussion indicates that SPPs are better confined when Fermi level is lower. So the Fermi level is selected to be $0.42 \mathrm{eV}, 0.43 \mathrm{eV}, 0.44 \mathrm{eV}, 0.45 \mathrm{eV}$, and $0.46 \mathrm{eV}$, respectively. The corresponding transmission spectra are denoted by blue, pink, green, purple, and red lines, respectively. As the Fermi level $E_{f}$ decreases, an obvious red shift of the transmission dip can be found. Obviously, the larger Fermi levels correspond to the higher transmission dips at the same resonance mode, shown in Figure 3(a). It is because that the loss of propagation mode determined by the imaginary part of $k_{\mathrm{SPP}}$ is inversely proportional to the Fermi level. 


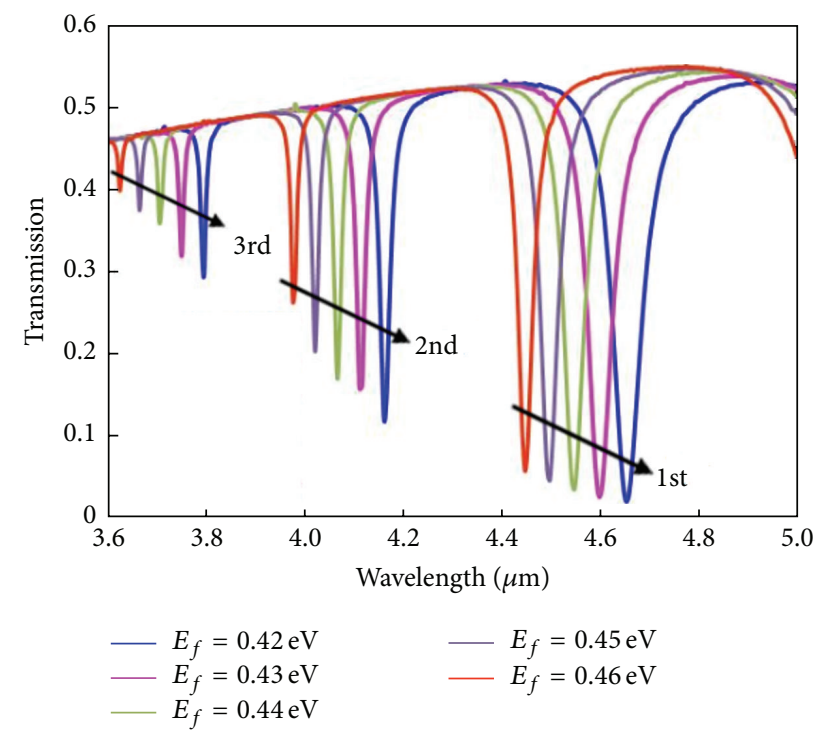

(a)

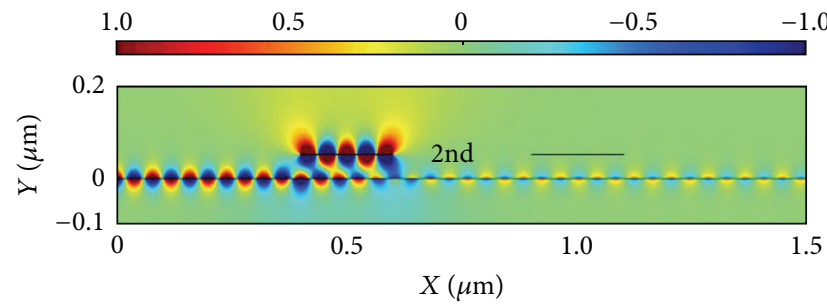

(c)

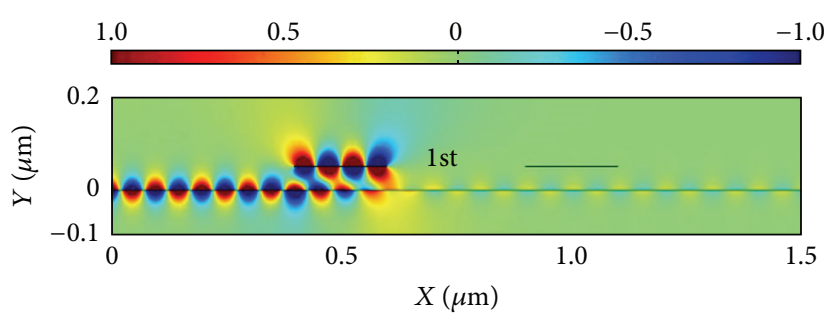

(b)

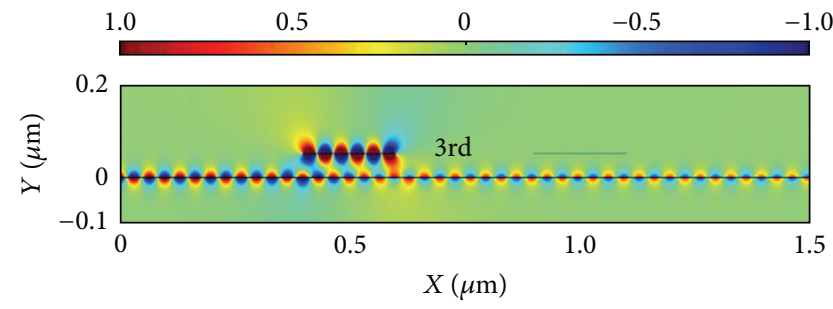

(d)

FIGURE 3: The graphene system with the right graphene sheet turned off. (a) The transmission spectra correspond to different Fermi level $E_{f}$ of left graphene sheet, $E_{f}=0.42 \mathrm{eV}, 0.43 \mathrm{eV}, 0.44 \mathrm{eV}, 0.45 \mathrm{eV}$, and $0.46 \mathrm{eV}$ denoted by blue, pink, green, purple, and red lines, respectively. The magnetic distributions $\left|H_{z}\right|$ correspond to different resonance wavelengths when the Fermi level has been fixed at $0.42 \mathrm{eV}$. (b) $\lambda_{r}=3.8 \mu \mathrm{m}$. (c) $\lambda_{r}=4.17 \mu \mathrm{m}$. (d) $\lambda_{r}=4.66 \mu \mathrm{m}$.

As shown in Figures 3(b)-3(d), when SPPs on graphene meets the resonant condition, the energy can be confined in graphene sheet by forming a standing wave mode, which leads transmission dips. According to the above analysis, this structure can achieve filter effects by controlling Fermi level without optimizing geometric parameters.

Next, the influence of interval $t$ has been investigated. The transmission spectra have been calculated for different values of $t(45 \mathrm{~nm}, 50 \mathrm{~nm}, 55 \mathrm{~nm}, 60 \mathrm{~nm}$, and $65 \mathrm{~nm})$, shown in Figure 4. The transmission spectra of $t$ selected to be $45,50,55,60$, and $65 \mathrm{~nm}$ are represented by blue, pink, green, purple, and red lines, respectively. Obviously, as the interval $t$ increases, the coupling process becomes weaker. In order to investigate the coupling between main waveguide and graphene sheet concretely, the coupled mode theory is introduced to interpret the simulation results. By using coupled mode theory, the transmission can be expressed as $T=\left(1 / \tau_{\omega}\right)^{2} /\left[\left(\omega-\omega_{0}\right)^{2}+\left(1 / \tau_{i}+1 / \tau_{\omega}\right)^{2}\right]$ [37]. Here, $\omega$ and $\omega_{0}$ are the incident frequency and the resonance frequency, respectively. $1 / \tau_{i}$ is the decay rate resulting from the intrinsic loss of graphene sheet resonator, and $1 / \tau_{\omega}$ is the decay rate due to the power escaping from the graphene waveguide. With the growing of interval $t$, the decay rate $1 / \tau_{\omega}$ decreases while the decay rate $1 / \tau_{i}$ remains the same. In this way, the energy confined in graphene resonator will become less for an increasing interval $t$, which leads to a growing value of the transmission dip. At the same time, the spectra width $\Delta \lambda \approx 4 \pi c\left(1 / \tau_{i}+1 / \tau_{\omega}\right) / \omega_{0}^{2}$ is proportional to the decay rate $1 / \tau_{\omega}$. That explains that the spectrum width becomes narrower with a growing interval $t$ at one resonance mode. This structure can achieve best filter effect when interval $t$ is fixed at $50 \mathrm{~nm}$.

In this section, the discussion focuses on the phase coupling between two graphene sheet resonators. To avoid the direct interference between two graphene sheets, the separation $l$ has been chosen to be large enough. The other geometric parameters are selected as follows: $l=300 \mathrm{~nm}$ and $t=50 \mathrm{~nm}$. We know that the resonance wavelength can be tuned via gate voltage on graphene sheets through the resonant condition. The difference of resonant wavelength between two graphene resonators is defined as $\delta_{i}=\lambda_{1}-$ $\lambda_{2}$ ( $i=1,2,3$ for each mode). The $E_{f}$ of left graphene sheet is fixed at $0.42 \mathrm{eV}$, which presents three resonance dips $(4.655 \mu \mathrm{m}, 4.165 \mu \mathrm{m}, 3.8 \mu \mathrm{m})$ corresponding to first-order, second-order, and third-order resonance modes depicted in Figure $3(\mathrm{a})$. The gradually increasing $\delta_{i}$ leads to a broader gap between two resonance dips for three resonance modes as $E_{f}$ of the right sheet grows from $0.425 \mathrm{eV}$ to $0.44 \mathrm{eV}$. In Figures 5(a), 5(b), and 5(c), for each resonance mode, all PIT windows 


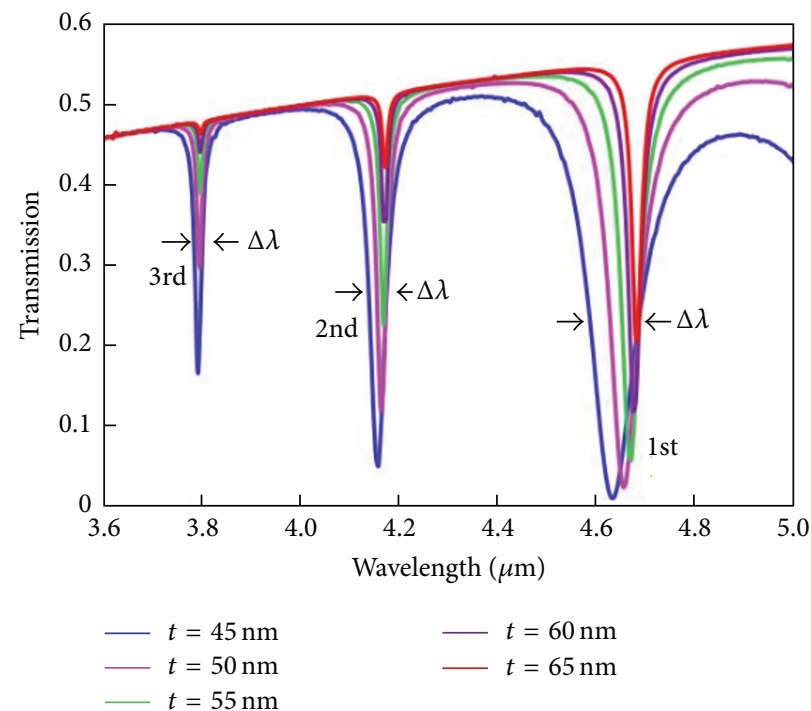

FIGURE 4: The graphene system with the right graphene sheet turned off. The transmission spectra correspond to different coupling interval $t$ of graphene sheets; $t=45 \mathrm{~nm}, 50 \mathrm{~nm}, 55 \mathrm{~nm}, 60 \mathrm{~nm}$, and $65 \mathrm{~nm}$ are expressed by blue, pink, green, purple, and red lines, respectively.

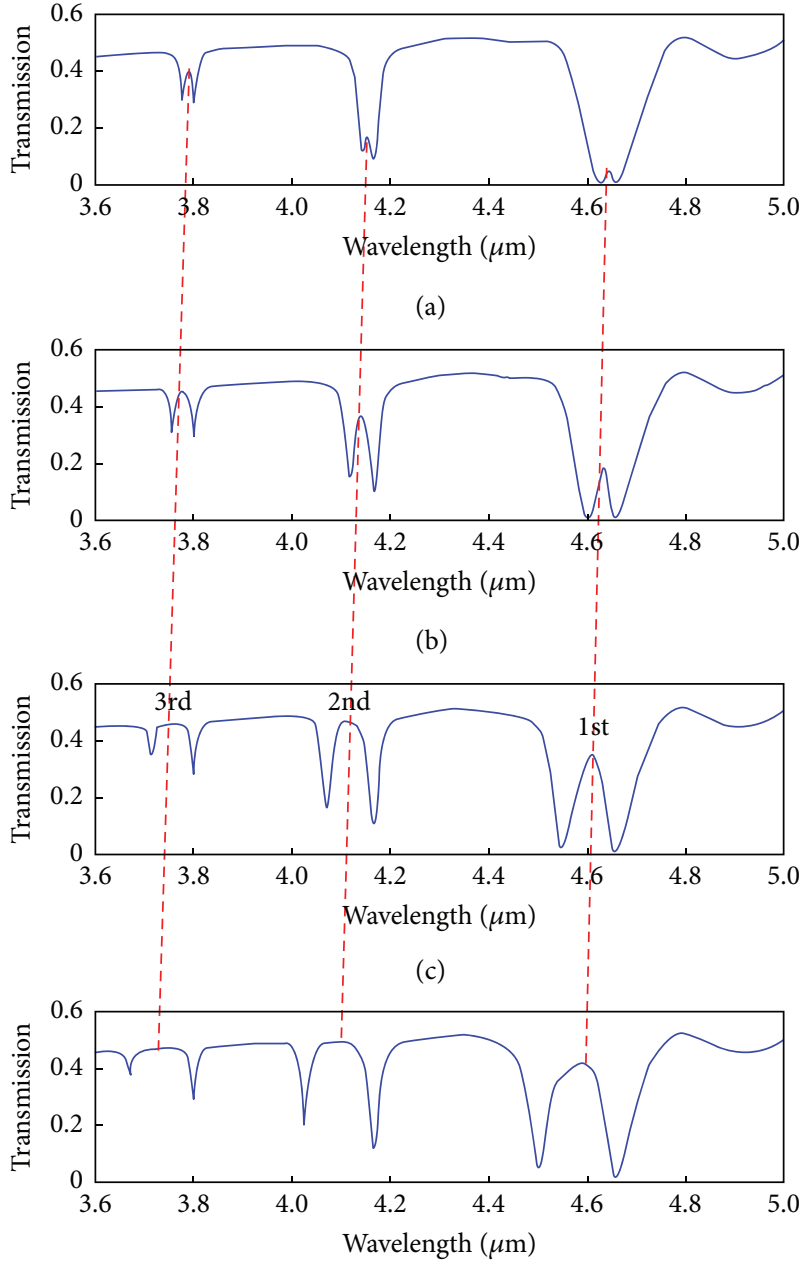

(d)

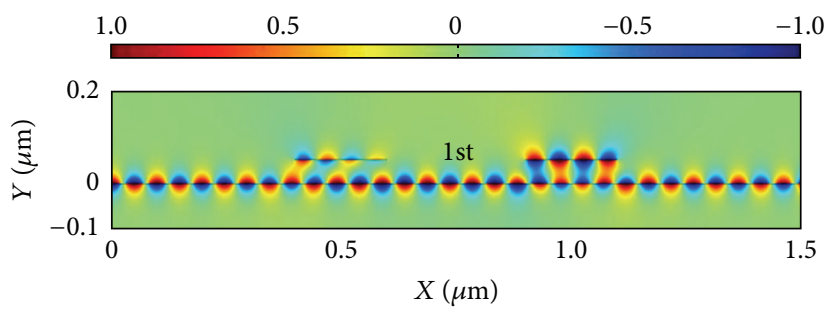

(e)

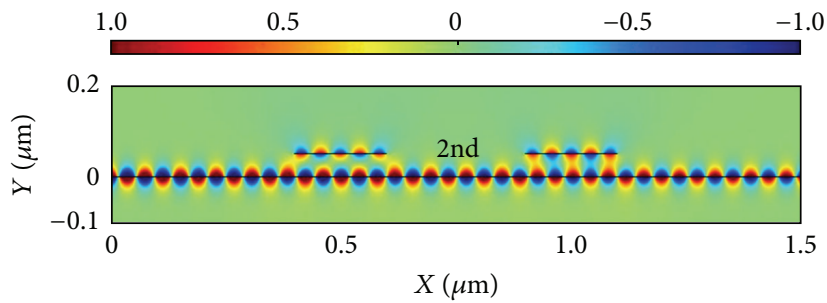

(f)

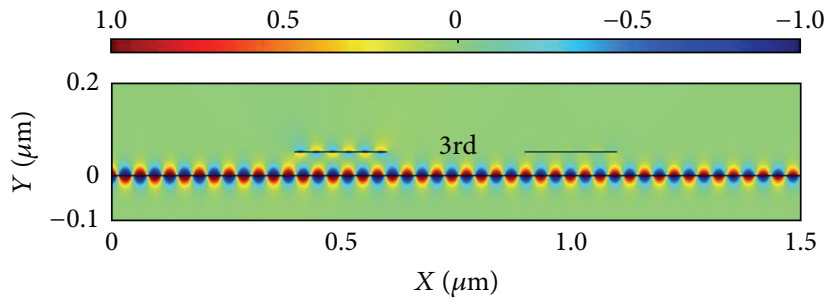

(g)

Figure 5: The Fermi level $E_{f}$ of left graphene sheet is fixed at $0.42 \mathrm{eV}$ and the Fermi level $E_{f}$ of right graphene sheet is set as follows: (a) $E_{f}=0.425 \mathrm{eV}$, (b) $E_{f}=0.43 \mathrm{eV}$, (c) $E_{f}=0.44 \mathrm{eV}$, and (d) $E_{f}=0.46 \mathrm{eV}$. The magnetic distributions $\left|H_{z}\right|$ corresponding to the transparency peaks. (e) $\lambda=4.61 \mu \mathrm{m}$ for $1 \mathrm{st}$ resonance mode. (f) $\lambda=4.11 \mu \mathrm{m}$ for $2 \mathrm{nd}$ resonance mode. (g) $\lambda=3.76 \mu \mathrm{m}$ for $3 \mathrm{rd}$ resonance mode. 


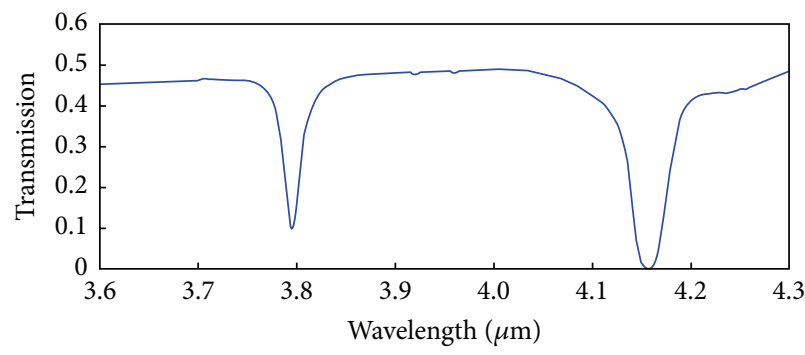

(a)

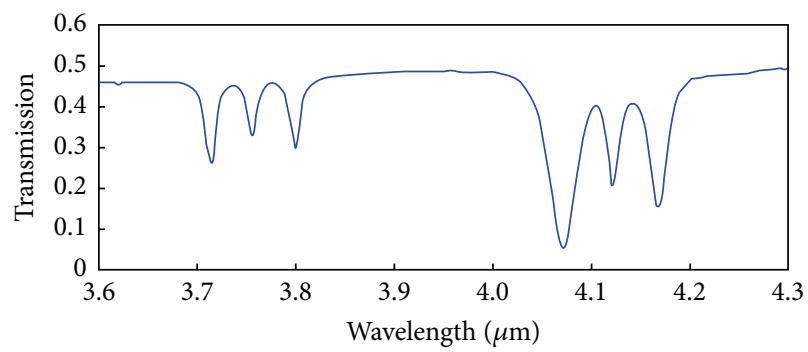

(c)

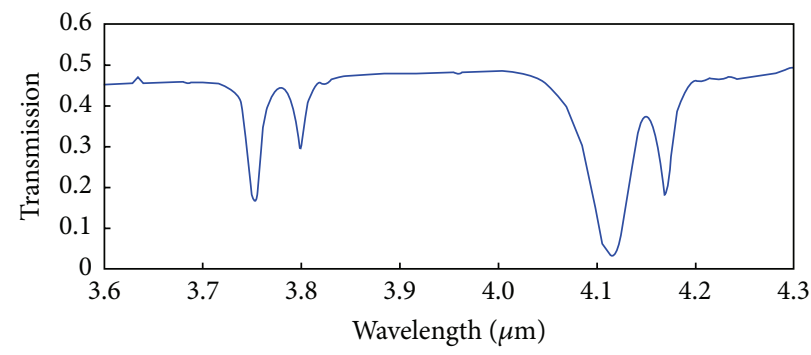

(b)

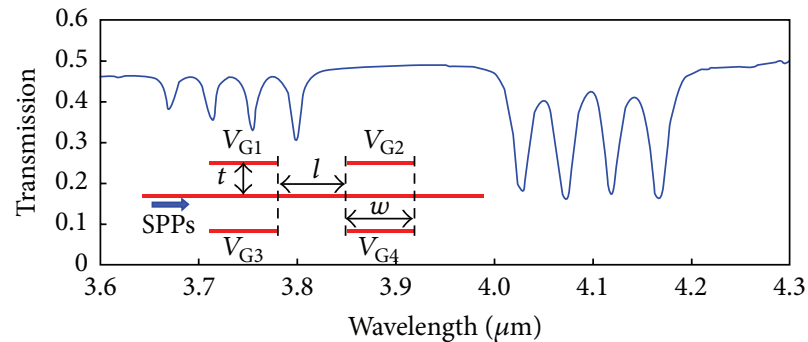

(d)

FIGURE 6: The transmission spectra of multipeak PIT. The Fermi level of graphene waveguide is fixed at $0.4 \mathrm{eV}$. (a) $E_{f 1}=E_{f 2}=E_{f 3}=E_{f 4}=$ $0.42 \mathrm{eV}$. (b) $E_{f 1}=E_{f 2}=0.42 \mathrm{eV}, E_{f 3}=E_{f 4}=0.43 \mathrm{eV}$. (c) $E_{f 1}=E_{f 2}=0.42 \mathrm{eV}, E_{f 3}=0.43 \mathrm{eV}$, and $E_{f 4}=0.44 \mathrm{eV}$. (d) $E_{f 1}=0.42 \mathrm{eV}$, $E_{f 2}=0.43 \mathrm{eV}, E_{f 3}=0.44 \mathrm{eV}$, and $E_{f 4}=0.45 \mathrm{eV}$.

show obvious off-to-on feature and the transparency bands become broader with the $\delta_{i}$ growing. The magnetic distributions $\left|H_{z}\right|$ corresponding to the transparency peaks of 1st, 2nd, and 3rd resonance modes are depicted in Figures 5(e), $5(\mathrm{f})$, and $5(\mathrm{~g})$. In this way, the tunable PIT effects based on graphene sheet resonators are achieved.

In this section, the phase coupling between the four graphene sheets structure has been investigated by contrasting with the two grapheme sheets structure. The extra graphene sheets are placed to graphene waveguide symmetric position on the basis of two sheets structure, which is shown in insert picture of Figure 6(d). The transmission spectra of second-order mode and third-order mode are depicted in Figures 6(a), 6(b), 6(c), and 6(d); the modulation of multipeak PIT can be achieved by controlling the Fermi level of four graphene sheets. By adjusting Fermi level gradually can result in tunable off-to-on multipeak PIT feature. Figure 7 shows the transmission spectra in the mid-infrared region of two peaks PIT based on the four graphene sheets structure. The upper and lower picture of Figure 7 show the magnetic distributions of the second-order mode under $\lambda_{0}=4.105 \mu \mathrm{m}, 4.12 \mu \mathrm{m}$, and $4.14 \mu \mathrm{m}$, which correspond to the standing wave modes originating from the splitting of the second-order resonance. In addition, Figure 8 shows the transmission spectra of three peaks PIT based on the four graphene sheets structure. The upper and lower picture of Figure 8 show the magnetic distributions of the second-order mode under $\lambda_{0}=4.05 \mu \mathrm{m}, 4.1 \mu \mathrm{m}$, and $4.12 \mu \mathrm{m}$. Due to the great difference of resonant wavelengths in first-order mode from Figure 3(a), the first-order mode of multipeak PIT has disorderly phenomenon because of the interference between two random resonance wavelengths.

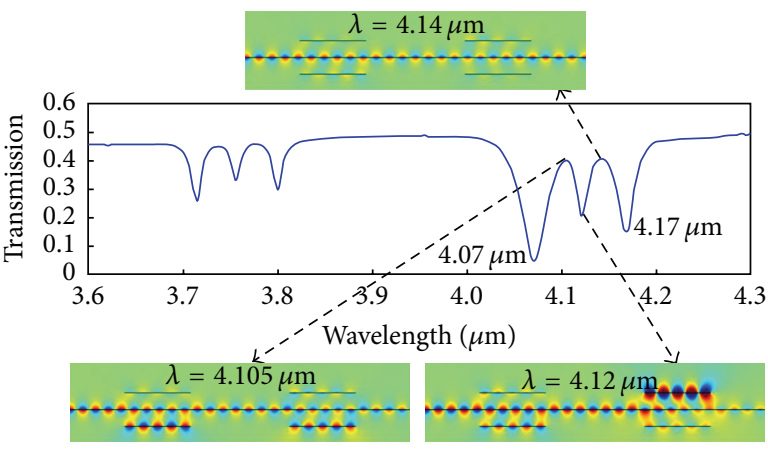

FIGURE 7: The transmission spectra of two-peak PIT based on the four graphene sheets structure. Magnetic field distributions of the 2nd mode under $\lambda_{0}=4.105 \mu \mathrm{m}, 4.12 \mu \mathrm{m}$, and $4.14 \mu \mathrm{m}$, respectively.

\section{Conclusion}

In this paper, we have numerically investigated a multimode plasmonically induced transparency (PIT) device operating in the mid-infrared region. The proposed model consisted of one graphene ribbon as main waveguide and two graphene sheets as resonators. In phase coupling systems, the simulation results evidently demonstrate the multimode PIT phenomenon. On the other hand, the multipeak PIT spectral responses emerge in four graphene sheets system. For phase coupling, the off-to-on PIT feature could be effectively achieved by varying the Fermi level of one graphene sheet resonator. Besides, by turning off one graphene resonator, this structure can achieve filter effects. The filter wavelength can be adjusted via varying the Fermi energy of graphene 


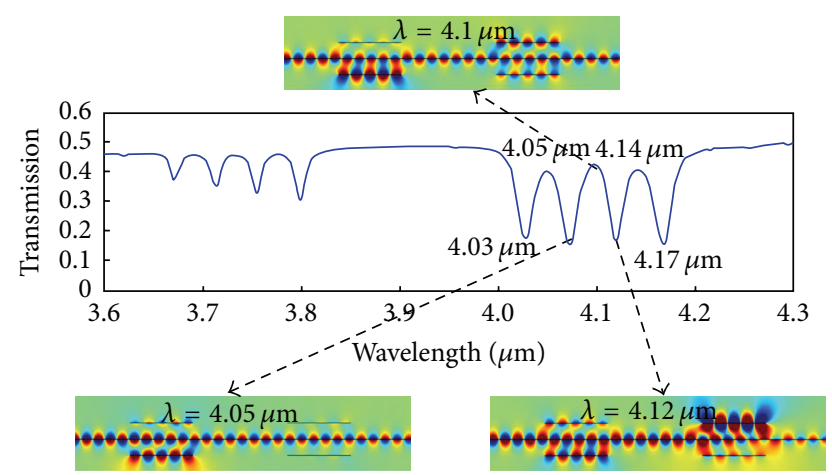

FIGURE 8: The transmission spectra of three-peak PIT based on the four graphene sheets structure. Magnetic distributions of the 2nd mode under $\lambda_{0}=4.05 \mu \mathrm{m}, 4.1 \mu \mathrm{m}$, and $4.12 \mu \mathrm{m}$, respectively.

resonator without optimizing geometric parameters. All of the observed PIT spectral features are of great importance to provide platforms for the design of the dynamical control PIT systems. The work may open up new ways for the development of compact thermal plasmonic devices such as tunable sensors, spectral splitter, and switchers in midinfrared region.

\section{Competing Interests}

The authors declare that they have no competing interests.

\section{Acknowledgments}

This work is supported by the National Basic Research Program of China (Grant no. 2013CBA01702), the National Natural Science Foundation of China (Grant no. 61377016, 61308017, and 11504139), and the Natural Science Foundation of Jiangsu Province (Grant no. BK20140167).

\section{References}

[1] P. Berini, "Plasmon-polariton waves guided by thin lossy metal films of finite width: bound modes of symmetric structures," Physical Review B, vol. 61, no. 15, pp. 10484-10503, 2000.

[2] W. L. Barnes, A. Dereux, and T. W. Ebbesen, "Surface plasmon subwavelength optics," Nature, vol. 424, no. 6950, pp. 824-830, 2003.

[3] K.-J. Boller, A. Imamolu, and S. E. Harris, "Observation of electromagnetically induced transparency," Physical Review Letters, vol. 66 , no. 20, pp. 2593-2596, 1991.

[4] S. E. Harris, "Electromagnetically induced transparency," Physics Today, vol. 50, no. 7, pp. 36-42, 1997.

[5] B. J. Tang, J. Wang, X. Xia, X. Liang, C. Song, and S. Qu, "Plasmonic-induced transparency and unidirectional control based on the waveguide structure with quadrant ring resonators," Applied Physics Express, vol. 8, no. 3, Article ID 032202, 2015.

[6] J. Wang, B. Yuan, C. Fan et al., "A novel planar metamaterial design for electromagnetically induced transparency and slow light," Optics Express, vol. 21, no. 21, pp. 25159-25166, 2013.

[7] T. F. Krauss, "Why do we need slow light?" Nature Photonics, vol. 2, no. 8, pp. 448-450, 2008.
[8] M. Fleischhauer, A. Imamoglu, and P. J. Marangos, "Electromagnetically induced transparency," Reviews of Modern Physics, vol. 77, no. 2, pp. 633-673, 2005.

[9] S. Zhang, D. A. Genov, Y. Wang, M. Liu, and X. Zhang, "Plasmon-induced transparency in metamaterials," Physical Review Letters, vol. 101, no. 4, Article ID 047401, 2008.

[10] N. Liu, L. Langguth, T. Weiss et al., "Plasmonic analogue of electromagnetically induced transparency at the Drude damping limit," Nature Materials, vol. 8, no. 9, pp. 758-762, 2009.

[11] J. Gu, R. Singh, X. Liu et al., "Active control of electromagnetically induced transparency analogue in terahertz metamaterials," Nature Communications, vol. 3, article 1151, 2012.

[12] Y. H. Guo, L. Yan, W. Pan et al., "Electromagnetically induced transparency (EIT)-like transmission in side-coupled complementary split-ring resonators," Optics Express, vol. 20, no. 22, pp. 24348-24355, 2012.

[13] X. Liu, J. Gu, R. Singh et al., "Electromagnetically induced transparency in terahertz plasmonic metamaterials via dual excitation pathways of the dark mode," Applied Physics Letters, vol. 100, no. 13, Article ID 131101, 2012.

[14] G. Cao, H. Li, S. Zhan et al., "Formation and evolution mechanisms of plasmon-induced transparency in MDM waveguide with two stub resonators," Optics Express, vol. 21, no. 8, pp. 91989205, 2013.

[15] X. Xia, J. Wang, F. Zhang et al., "Multi-mode plasmonically induced transparency in dual coupled graphene-integrated ring resonators," Plasmonics, vol. 10, no. 6, pp. 1409-1415, 2015.

[16] X. Wang, X. Xia, J. Wang, F. Zhang, Z. Hu, and C. Liu, "Tunable plasmonically induced transparency with unsymmetrical graphene-ring resonators," Journal of Applied Physics, vol. 118, no. 1, Article ID 013101, 2015.

[17] X. Y. Duan, S. Chen, H. Cheng, Z. Li, and J. Tian, "Dynamically tunable plasmonically induced transparency by planar hybrid metamaterial," Optics Letters, vol. 38, no. 4, pp. 483-485, 2013.

[18] A. H. Castro Neto, F. Guinea, N. M. R. Peres, K. S. Novoselov, and A. K. Geim, "The electronic properties of graphene," Reviews of Modern Physics, vol. 81, no. 1, pp. 109-162, 2009.

[19] L. Ju, B. Geng, J. Horng et al., "Graphene plasmonics for tunable terahertz metamaterials," Nature Nanotechnology, vol. 6, no. 10, pp. 630-634, 2010.

[20] B. Wang, X. Zhang, X. Yuan, and J. Teng, "Optical coupling of surface plasmons between graphene sheets," Applied Physics Letters, vol. 100, Article ID 131111, 2012.

[21] B. Wang, X. Zhang, F. J. García-Vidal, X. Yuan, and J. Teng, "Strong coupling of surface plasmon polaritons in monolayer graphene sheet arrays," Physical Review Letters, vol. 109, no. 7, Article ID 073901, 2012.

[22] A. K. Geim, "Graphene: status and prospects," Science, vol. 324, no. 5934, pp. 1530-1534, 2009.

[23] Q. Bao, H. Zhang, B. Wang et al., "Broadband graphene polarizer," Nature Photonics, vol. 5, no. 7, pp. 411-415, 2011.

[24] M. Liu, X. Yin, E. Ulin-Avila et al., "A graphene-based broadband optical modulator," Nature, vol. 474, no. 7349, pp. 64-67, 2011.

[25] S. Thongrattanasiri, F. H. L. Koppens, and F. J. García de Abajo, "Complete optical absorption in periodically patterned graphene," Physical Review Letters, vol. 108, no. 4, Article ID 047401, 2012.

[26] A. Vakil and N. Engheta, "Transformation optics using graphene," Science, vol. 332, no. 6035, pp. 1291-1294, 2011. 
[27] C. Zeng, J. Guo, and X. Liu, "High-contrast electro-optic modulation of spatial light induced by graphene-integrated FabryPérot microcavity," Applied Physics Letters, vol. 105, Article ID 121103, 2014.

[28] X. Shi, D. Han, Y. Dai et al., "Plasmonic analog of electromagnetically induced transparency in nanostructure graphene," Optics Express, vol. 21, no. 23, pp. 28438-28443, 2013.

[29] P.-Y. Chen and A. Alù, "Atomically thin surface cloak using graphene monolayers," ACS Nano, vol. 5, no. 7, pp. 5855-5863, 2011.

[30] G. W. Hanson, "Quasi-transverse electromagnetic modes supported by a graphene parallel-plate waveguide," Journal of Applied Physics, vol. 104, no. 8, Article ID 084314, 2008.

[31] K. S. Novoselov, A. K. Geim, S. V. Morozov et al., "Electric field effect in atomically thin carbon films," Science, vol. 306, no. 5696, pp. 666-669, 2004.

[32] H.-S. Chu and C. How Gan, "Active plasmonic switching at midinfrared wavelengths with graphene ribbon arrays," Applied Physics Letters, vol. 102, no. 23, Article ID 231107, 2013.

[33] J. Christensen, A. Manjavacas, S. Thongrattanasiri, F. H. L. Koppens, and F. J. García De Abajo, "Graphene plasmon waveguiding and hybridization in individual and paired nanoribbons," ACS Nano, vol. 6, no. 1, pp. 431-440, 2012.

[34] H. Lu, "Plasmonic characteristics in nanoscale graphene resonator-coupled waveguides," Applied Physics B, vol. 118, no. 1, pp. 61-67, 2015.

[35] J. L. Garcia-Pomar, A. Y. Nikitin, and L. Martin-Moreno, "Scattering of graphene plasmons by defects in the graphene sheet," ACS Nano, vol. 7, no. 6, pp. 4988-4994, 2013.

[36] A. Y. Nikitin, T. Low, and L. Martin-Moreno, "Anomalous reflection phase of graphene plasmons and its influence on resonators," Physical Review B, vol. 90, Article ID 041407, 2014.

[37] Q. Li, T. Wang, Y. Su, M. Yan, and M. Qiu, "Coupled mode theory analysis of mode-splitting in coupled cavity system," Optics Express, vol. 18, no. 8, pp. 8367-8382, 2010. 

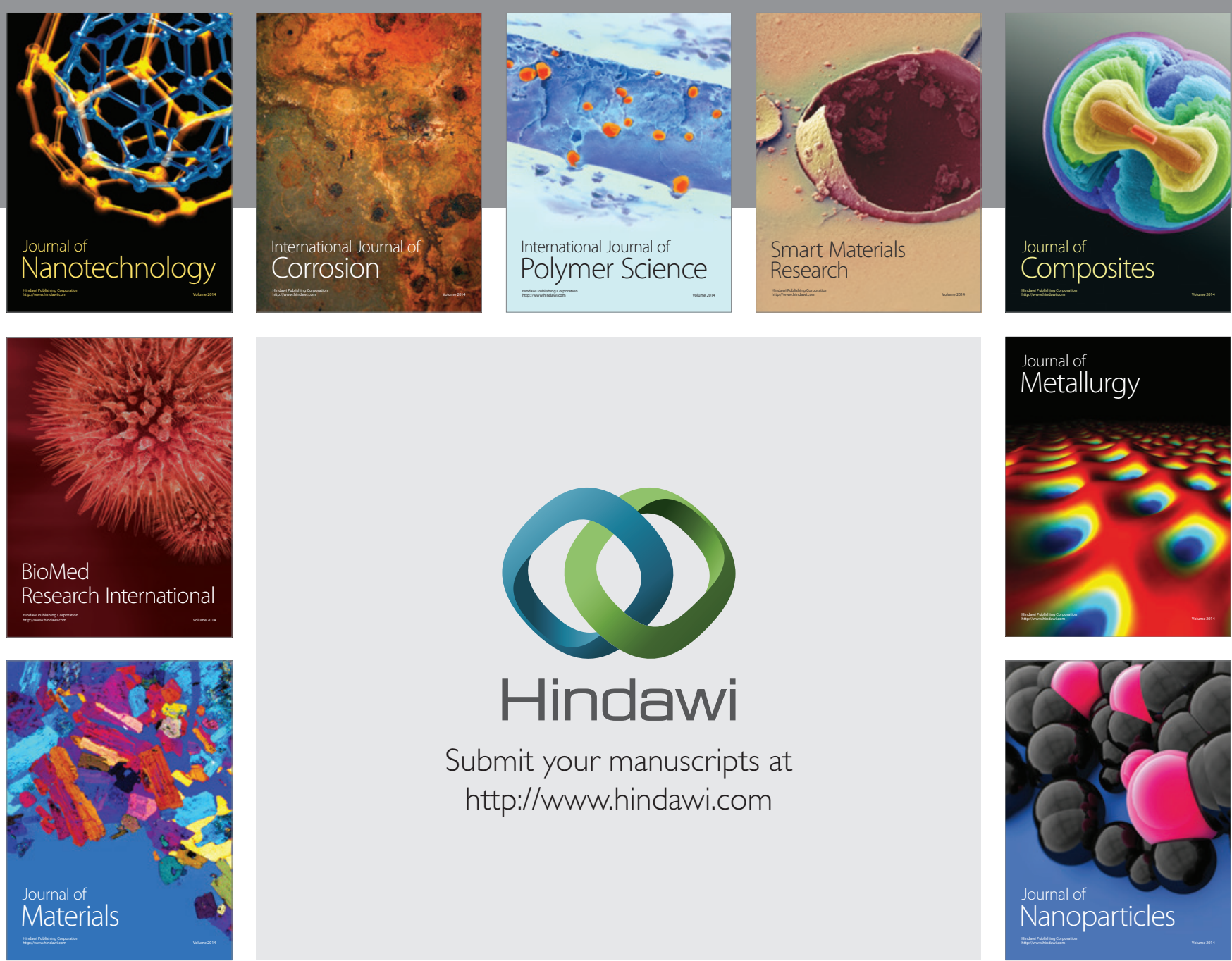

\section{Hindawi}

Submit your manuscripts at

http://www.hindawi.com

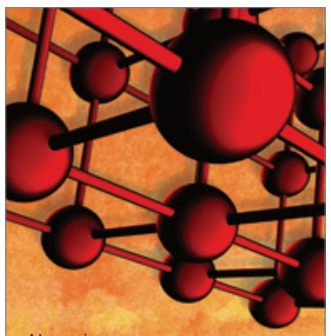

Materials Science and Engineering
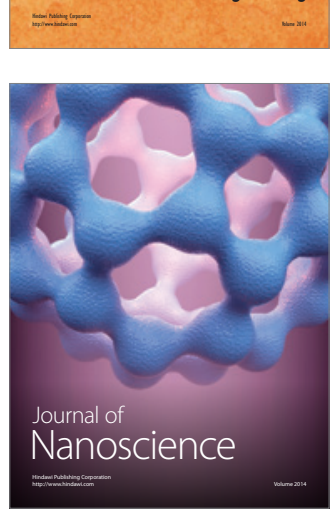
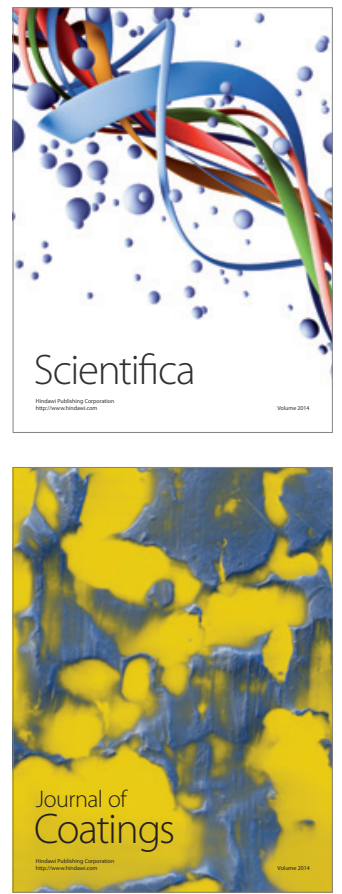
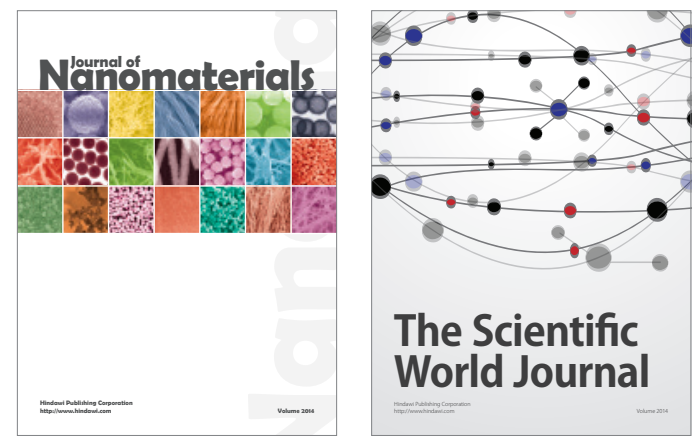

The Scientific World Journal
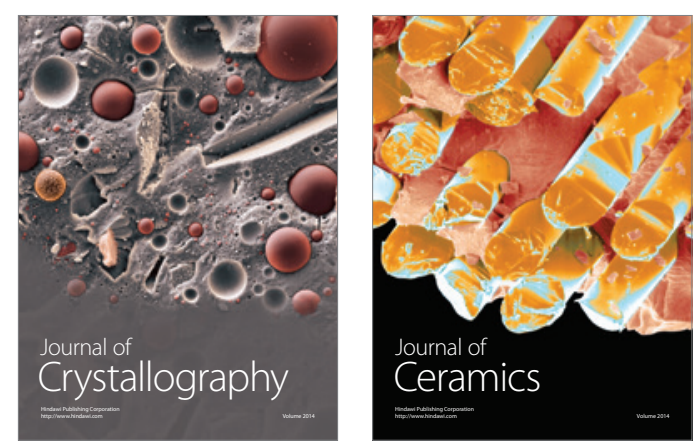
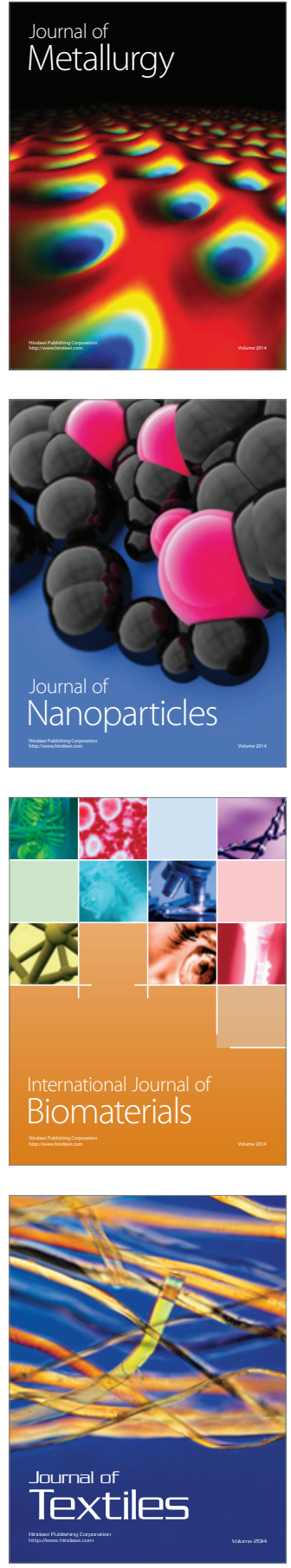